\title{
THE USE OF RELATED VARIABLES IN EXPLAINING GENOTYPE-ENVIRONMENT INTERACTIONS
}

\author{
G. H. FREEMAN and P. CRISP \\ National Vegetable Research Station, Wellesbourne, Warwick
}

Received 19.vi.78

\begin{abstract}
SUMMARY
When genotypes are grown in a range of environments several variables are often recorded on the same genotype. Regressing one character on another may not only give useful information about the relation between them but also help to explain genotype-environment interactions observed in the character of primary interest. Three examples are given in which weight or height is related to a measure of chronological age for a series of cauliflower, lettuce and Nicotiana trials. The conclusions drawn from this technique may be of practical plant breeding importance and also illustrate how the method may be used to assist the interpretation of genotype-environment interactions.
\end{abstract}

\section{INTRODUCTION}

IN many trials in which a set of plant genotypes is grown over a range of environments the genotypes do not behave in the same relative way in all environments. This phenomenon is known as genotype-environment (GE) interaction, and many methods have been proposed for its statistical analysis, these having been reviewed critically by Freeman (1973) and Hill (1975). The values taken by the individual genotypes in the set of environments are often linearly related to the mean value of all genotypes in these environments. A regression method to deal with this situation was introduced by Yates and Cochran (1938), and this technique is now usually known as joint regression analysis. Joint regression analysis has been used in much subsequent work (e.g. Finlay and Wilkinson, 1963; Perkins and Jinks, 1968), and although there have been statistical objections to the technique (e.g. Knight, 1970; Freeman, 1973) it has frequently been found to give a very satisfactory description of observed biological phenomena. In particular, genotypes which vary comparatively little in different environments and so have regression coefficients of less than unity are regarded as stable, and these may be of value to the plant breeder.

Alternative measures of the environment have been sought, either the responses of similar genotypes (Fripp and Caten, 1971; Freeman and Perkins, 1971), or both biological and physical measures (Fripp, 1972; Perkins and Jinks, 1973), but basically similar regression procedures were used to analyse the data. Rather different statistical models for regression on environmental variables were considered by Hardwick and Wood (1972) and Wood (1976).

The factor common to all the work so far described is that only one variable is considered for each genotype in an environment no matter how many measures of the environment are taken. Often in practice, however, several variables are measured on each phenotype. Partitioning phenotypic correlations between variables into genotypic and environmental correlations may indicate the effects that selection for one variable may have

$$
42 / 1-\mathrm{A}
$$


on another, and the effects that different environments have on the correlated expression of these variables (e.g. Dowker et al., 1976). Another technique is to combine these correlations to give an overall selection index which can be used by the breeder, but this index may be unsatisfactory if there are substantial GE interactions (e.g. Arnold and Innes, 1975).

A complementary approach is to try to explain the variation in one variable in terms of variation in one or more others. The analysis of covariance is frequently used to account for variation in a dependent variable $y$ by means of differences in an independent variable $x$. In the commonest use of this technique the variable $x$ is unaffected by any treatments that may be applied to $y$. However, as pointed out by Cochran (1957), one important use is to throw light on the nature of treatment effects in the situation where $x$ and $y$ are both affected by the treatments. A similar argument holds when considering two related variables measured on a set of genotypes in a range of environments in order to explain GE interaction. Thus, Crisp et al. (1977), in studying resistance in radish to cabbage root fly, found that in some cases use of the changing attractiveness of the plants over time as a covariate reduced GE interactions but without affecting varietal differences.

However, the relationship between two variables may not be amenable to analysis of covariance. If they both display GE interaction, the relation between them over the range of environments may differ between genotypes. Only if this relation is the same is it possible to use analysis of covariance to see if the GE interaction in one variable $y$ is explained by the GE interaction in a related variable $x$.

We describe here the statistical principles of the analysis of related variables within genotypes and the GE interaction. We illustrate this with examples and show where the interpretation of GE interaction has been assisted by the technique, and has in one case suggested an efficient method of breeding for stable performance.

\section{STATISTICAL RELATIONSHIPS BETWEEN CORRELATED VARIABLES}

If two variables $x$ and $y$ display GE interaction, is the relation between them over the range of environments the same for each genotype? Only if this is true is it possible to use analysis of covariance to answer a second, more important question: is the interaction in $y$ entirely due to its relation with $x$ ?

If the relation between the variables $y$ and $x$ depends on the genotype, then, for the $i$ th genotype, $j$ th environment and $k$ th replicate $y_{i j k}=f_{i}\left(x_{i j k}\right)+$ $e_{i j k}$, where $f_{i}$ is some function of $x$ and $e_{i j k}$ is random variation. This is a general relation between $y$ and $x$, and may be modified to allow for the previous removal of the effects on $y$ and $x$ of genotypes, environments or both. This functional relation, however accurate, will seldom be resolvable unless it can be expressed in a reasonably simple form. A linear, or possibly a quadratic, regression is usually all that can be managed in practice, an appropriate quadratic regression to describe the relation within the $i$ th genotype being:

$$
y_{i j k}=a_{i 0}+a_{i 1} x_{i j k}+a_{i 2} x_{i j k}^{2}+e_{i j k} .
$$

It is easier to separate out the constant, linear and quadratic terms in this relation if orthogonal polynomials are used, that is:

$$
y_{i j k}=b_{i 0}+b_{i 1}\left(x_{i j k}-\bar{x}_{i}\right)+b_{i 2}\left(x_{i j k}^{2}-p_{i} x_{i j k}-q_{i}\right)+e_{i j k}
$$


In equation ( $I) b_{i 0}$ and $\bar{x}_{i}$ are the mean values of $y$ and $x$ respectively for the $i$ th genotype, and $p_{i}, q_{i}$ are such that the estimators $b_{i 0}, b_{i 1}$ and $b_{i 2}$ are uncorrelated. $b_{i 1}$ is the regression coefficient for the linear regression of of $y$ on $x$ for the $i$ th genotype, and $b_{i 2}$ the regression coefficient for the quadratic term in the corresponding quadratic regression. The value of $b_{i \mathbf{1}}$ is the same as that obtained if linear regression only is conside $e_{\iota}$. The full expressions for $p_{i}$ and $q_{i}$ ure too complicated to give here but are given in standard statistical textbooks, for example Kendall and Stuart (1973).

The corresponding expression for regression on $z_{i j k}$ where $z_{i j k}$ is $x_{i j k}$ adjusted for both genotypic and environmental effects is:

$$
y_{i j k}=\mu+d_{i}+\epsilon_{j}+c_{i 1} z_{i j k}+c_{i 2}\left(z_{i j k}{ }^{2}-p_{i}{ }^{\prime} z_{i j k}-q_{i}{ }^{\prime}\right)+e_{i j k}
$$

In equation (2) $\mu$ is the mean of $y$ for all genotypes, and $d_{i}$ and $\epsilon_{j}$ are respectively genotypic and environmental effects for $y$. If the relations are linear rather than quadratic, $b_{i 2}$ and $c_{i 2}=0$ and equations (1) and (2) simplify. If, and only if, the values of $b_{i 1}, b_{i 2}$ or $c_{i 1}, c_{i 2}$ are independent of genotype is it possible to use analysis of covariance to compare genotypic effects. In practice both main effects and interactions in the related variables $y$ and $x$ may well show different patterns, and then it would be unlikely that the $b$ 's and $c$ 's are independent of genotype. The use of more than one $x$ variable can be described by an extension of (1) and (2); interpretation of the results, though raising no new points of principle, may become more difficult in practice.

There is an important difference between equations (1) and (2). For the $i$ th genotype equation ( $I$ ) is concerned with explaining the variation in one variable $y$ accounted for by a related variable $x$ reflecting environmental differences. Although inferences can be made from this relation which may be of use in interpreting GE interactions in $y$, equation (1) does not assess the effects of GE interaction on the relation between the two variables. The explanation of GE interaction is attempted in equation (2), where the overall effects of both genotype and environment have been removed: thus, equation (2) yields regression coefficients for each genotype which are independent of both genotypic and environmental effects, and any remaining relation between the two variables is due to GE interaction in $x$.

A different relation is postulated in joint regression analysis, namely:

$$
y_{i j k}=\mu+d_{i}+\epsilon_{j}+g_{i j}+e_{i j k}
$$

where $g_{i j}=\beta_{i} \epsilon_{j}+\delta_{i j}$, so that:

$$
y_{i j k}=\mu+d_{i}+\left(\mathrm{I}+\beta_{i}\right) \epsilon_{j}+\delta_{i j}+e_{i j k}
$$

In equation (3) $\mu, d_{i}$ and $\epsilon_{j}$ are the same as in equation (2) and $g_{i j}$ is an interaction effect which is regressed on $\epsilon_{j}$ with regression coefficient $\beta_{i}$ and deviation $\delta_{i j}$.

The $\beta_{i}$ in equation (3) is not the same as any of the regression coefficients in equations (1) and (2), for $\beta_{i}$ represents regression on the environmental mean of $y$, and the $b$ 's and $c$ 's regression on the related variable $x$. Further, it must be recognised that the method of joint regression analysis depends critically not only on the set of environments considered but also on the set of genotypes, because the value of $\beta_{i}$, unlike the values of the $b$ 's in equation (I), is influenced by genotypes other than the $i$ th. 
Equations (1), (2) and (3) thus summarise the analysis of data showing GE interactions in different ways, though, if only one variable is measured, regression such as (1) or (2) is not possible. When there are two related variables, one way of drawing more information from the data in order to account for genotypic differences and GE interactions is to correlate regression coefficients derived from the different equations. Correlations between the regression coefficients from equations (1) and (2) might occur if there were no overall environmental effect, but this is unlikely in practice. However, correlations between coefficients from equations (1) and (2) on the one hand and (3) on the other may give a greater understanding of the results obtained by joint regression analysis.

\section{GE INTERAGTION IN GAULIFLOWERS}

Kesavan et al. (1976) described the effects of genotype, environment and GE interaction on time from sowing to maturity (expressed as the logarithm) in a trial of autumn-maturing cauliflowers. Crisp and Kesavan (1978) described the results for curd weight at maturity in the same trial. The trial consisted of 12 genotypes grown in 36 environments. There was significant GE interaction for both variables: 10 per cent of the total components of variance for chronological age at maturity, and 42 per cent for curd weight. There was significant heterogeneity of regression for both variables in the joint regressions. Neither genotypic nor environmental effects as expressed by analysis of variance or joint regression were the same for these two variables, although they are commonly assumed to be related. We attempt here to explain the large GE interaction for curd weight in terms of the age at maturity, using untransformed data.

The relation between curd weight and age was found to be quadratic in this trial. That is, curds maturing after a short time were small, the curd weight rose to a maximum with an intermediate time to maturity, and was then smaller again as the time to maturity increased. A significant pattern of this kind was found over all genotypes together using equations (1) and (2), and for seven of the 12 genotypes using equation (1), and for four genotypes using equation (2). The coefficients derived for each genotype from equations (1) and (2) or their linear equivalents are shown in table 1, together with coefficients from the joint regressions for curd weight and age at maturity, that is, from equation (3).

Correlations between these coefficients revealed that $\beta_{w}$, the joint regression coefficient for curd weight, was negatively correlated with $b_{2}$ from equation (1) (table 2). For each of the 12 genotypes, values estimated from the quadratic and linear forms of equation (1) deviated from each other most at the greatest age at maturity. That is, $b_{2}$ from equation (1) had the greatest effects on plants which took a relatively long time to reach maturity. Therefore, the correlation between $\beta_{w}$ and $b_{2}$ may be interpreted as follows. Genotypes which were sensitive to the environment, as regards curd weight assessed by joint regression, also tended to show the most marked reduction in curd weight if they matured at a later chronological age.

This result is of potential importance to the breeder who is attempting to produce varieties of cauliflower with stable yields. He has previously been obliged to produce varieties and then screen these finished products for the required stability of curd weight (Crisp and Kesavan, 1978). The 
TABLE 1

Coefficients from the regressions of curd weight on chronological age at maturity in 12 cauliflower genotypes, and from the joint regressions of curd weight $(\mathrm{w})$ and age at maturity $(\mathrm{m})$

\begin{tabular}{|c|c|c|c|c|c|c|c|}
\hline \multirow[b]{3}{*}{ Genotype } & \multirow{3}{*}{$\begin{array}{c}\text { Mean } \\
\text { curd } \\
\text { weight } \\
b_{0}\end{array}$} & \multicolumn{4}{|c|}{$\begin{array}{l}\text { Kegressions or curd welgnt on age } \\
\text { at maturity }\end{array}$} & \multirow{2}{*}{\multicolumn{2}{|c|}{$\begin{array}{c}\text { Joint } \\
\text { regressions } \\
\text { Equation (3) }\end{array}$}} \\
\hline & & \multicolumn{2}{|c|}{ Equation (1) } & \multicolumn{2}{|c|}{ Equation (2) } & & \\
\hline & & $b_{1}$ & $b_{2}$ & $c_{1}$ & $c_{2}$ & $\beta_{w}$ & $\beta_{m}$ \\
\hline Le Cerf B Autumn & $277 \cdot 6$ & $-0 \cdot 608$ & -0.0121 & 0.679 & 0.0089 & -0.232 & 0.030 \\
\hline Lero & 187.8 & $-0 \cdot 116$ & -0.0104 & 1.068 & -0.0107 & -0.146 & -1.010 \\
\hline Le Cerf Improved & 196.9 & $0 \cdot 155$ & 0.0090 & 3.033 & 0.0571 & -0.618 & $-0 \cdot 104$ \\
\hline South Pacific & $180 \cdot 8$ & $-0 \cdot 189$ & -0.0407 & $1 \cdot 169$ & $-0 \cdot 1506$ & $0 \cdot 198$ & 0.149 \\
\hline Boomerang & $131 \cdot 2$ & 0.079 & 0.0101 & -0.095 & 0.0170 & -0.728 & -0.027 \\
\hline Kangaroo & $221 \cdot 0$ & $-0 \cdot 324$ & -0.0582 & 1.678 & -0.0718 & 0.363 & $0 \cdot 165$ \\
\hline Clandonian & $318 \cdot 5$ & -0.846 & -0.0648 & -0.284 & -0.0541 & 0.066 & -0.076 \\
\hline Hylite & $311 \cdot 0$ & $-0 \cdot 325$ & -0.0400 & 2.391 & $0 \cdot 2974$ & 0.361 & -0.066 \\
\hline Autumn Glory & $328 \cdot 4$ & -0.373 & -0.0239 & 0.479 & 0.2223 & 0.232 & -0.049 \\
\hline $\mathrm{KC}$ & $209 \cdot 6$ & -1.444 & -0.0384 & 1.788 & $-0 \cdot 3423$ & 0.095 & -0.095 \\
\hline YCM2 & $229 \cdot 4$ & $-0 \cdot 641$ & -0.0316 & $-4 \cdot 497$ & $-0 \cdot 0802$ & -0.053 & -0.026 \\
\hline AGAQ & $163 \cdot 1$ & -0.135 & -0.0392 & $3 \cdot 246$ & $-0 \cdot 1986$ & 0.266 & $0 \cdot 108$ \\
\hline
\end{tabular}

Regressions of curd weight on age

TABLE 2

Correlations between coefficients from the regressions of curd weight in 12 cauliflower genotypes

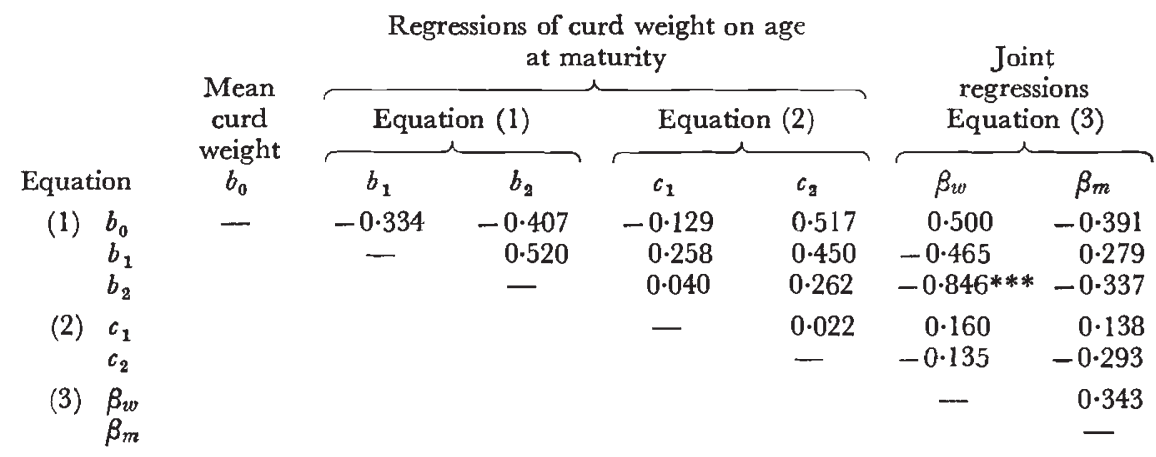

results of the analysis presented here suggest that he should extend the length of the growing period of a segregating population by appropriate seedbed and planting techniques such that curd weight is generally reduced. $\mathrm{He}$ should then select the plants with the largest curds to give the required stability of large curd weight in the resulting variety.

\section{GE INTERAGTION IN OUTDOOR LETTUCE}

An orthogonal set of data was made available to us by our colleague Dr D. Gray. The data were derived from 13 lettuce varieties which had been grown in eight field environments consisting of different seasons and sowing times. Analysis of variance revealed GE interaction for time from sowing to maturity and for head weight at maturity, with significant heterogeneity of regression in the joint regressions for head weight (table 3 ). 
The GE interaction components of variance were 2 per cent for chronological age at maturity, and 24 per cent for head weight at maturity. The regressions were calculated from equations (1), (2), and (3), using age as the independent variable, as described for cauliflower in the previous section. There were significant correlations between the head weight joint regression coefficients $\left(\beta_{w}\right)$ and $b_{0}$ and each of the coefficients of equation (1) (table 4). However, the only significant partial correlation between $\beta_{w}$ and any of the regression coefficients was that between $\beta_{w}$ and $b_{0}$, the mean head weight, eliminating $b_{1}$ and $b_{2}$, with a value of 0.645 $(\mathrm{P}<0 \cdot 05)$. That is, greater head weight was correlated with greater sensitivity to the environment for head weight, as measured by joint regression.

TABLE 3

Analysis of variance of chronological age at maturity and head weight at maturity in lettuce

Item

Environment (E)

Blocks within $\mathrm{E}$

Varieties (V)

$\mathrm{V} \times \mathrm{E}$

Joint regression of $\mathrm{V} \times \mathrm{E}$ :

Heterogeneity of regression

Deviations from regression

Error

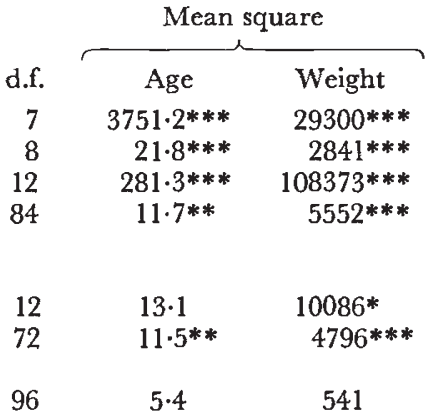

This kind of correlation is common in crop plants, where the best yielding genotypes are often also the least stable as measured by joint regression analysis. The opposite relations may also occur (see, for example, Gooding et al., 1975) and, as Brumpton et al. (1977) have shown, it may be possible to recombine high yield with high stability. The use of equation (1) has shown that $\beta_{w}$ is directly related to $b_{0}$ : the apparent relations between $\beta_{w}$ and either $b_{1}$ or $b_{2}$ shown in table 4 are found to be due entirely to their correlations with $b_{0}$. It is possible that correlations between high sensitivity and high yield in other crops are due in fact to their co-dependence on an unrelated variable (such as length of growing period). If this were the case, then instead of attempting to recombine high yield and low sensitivity per se into a single genotype, the breeder might be better advised to achieve this same end by recombining high yield with a particular type of response to the environment in terms of the unrelated variable, as we have advocated for the cauliflower.

\section{GE interaction IN NIGOtIANA}

Perkins and Jinks (1973) grew 10 inbred lines of Nicotiana rustica in soils treated with combinations of the mineral nutrients $\mathrm{N}, \mathrm{P}, \mathrm{K}$, and $\mathrm{Ca}$ to give 16 environments. Variables recorded included the age at flowering and final height. Analysis of variance showed that a significant proportion of the variance ( 4 per cent of the total) was ascribable to GE interaction for 
TABLE 4

Correlations between coefficients from the regressions of head weight in 13 lettuce genotypes

\begin{tabular}{|c|c|c|c|c|c|c|c|}
\hline \multirow[b]{3}{*}{ Equation } & \multirow{3}{*}{$\begin{array}{c}\text { Mean } \\
\text { head } \\
\text { weight } \\
b_{0}\end{array}$} & \multicolumn{4}{|c|}{$\begin{array}{l}\text { Regressions of head weight } \\
\text { on age at maturity }\end{array}$} & \multirow{2}{*}{\multicolumn{2}{|c|}{$\begin{array}{c}\text { Joint } \\
\text { regressions } \\
\text { Equation (3) }\end{array}$}} \\
\hline & & \multicolumn{2}{|c|}{ Equation (1) } & \multicolumn{2}{|c|}{ Equation (2) } & & \\
\hline & & $b_{1}$ & $b_{2}$ & $c_{1}$ & $c_{2}$ & $\beta_{w}$ & $\beta_{m}$ \\
\hline (1) $\begin{array}{l}b_{0} \\
b_{1} \\
b_{2}\end{array}$ & - & $\begin{array}{l}0 \cdot 686^{* *} \\
-\end{array}$ & $\begin{array}{l}0.923 * * * \\
0.649 * \\
-\end{array}$ & $\begin{array}{r}-0.030 \\
0.142 \\
0.077\end{array}$ & $\begin{array}{r}-0.022 \\
-0.404 \\
0.050\end{array}$ & $\begin{array}{l}0.920 * * * \\
0 \cdot 716^{* *} \\
0.843^{* * *}\end{array}$ & $\begin{array}{r}-0.085 \\
-0.099 \\
0.009\end{array}$ \\
\hline (2) $\begin{array}{ll}c_{1} \\
& c_{2}\end{array}$ & & & & - & $\begin{array}{l}0.068 \\
-\end{array}$ & $\begin{array}{r}0 \cdot 067 \\
-0 \cdot 283\end{array}$ & $\begin{array}{l}0 \cdot 101 \\
0.567\end{array}$ \\
\hline (3) $\begin{array}{l}\beta_{w} \\
\beta_{m}\end{array}$ & & & & & & - & -0.200 \\
\hline
\end{tabular}

each of these variables, and that there was a significant heterogeneity of regression from the joint regression for both variables. In an attempt to explain the GE interaction for height in terms of age at flowering we regressed the former on the latter. Three of the ro genotypes gave significant linear or quadratic regressions when equation (1) was used, and two when equation (2) was used. Correlation of the coefficients from these equations with those from the joint regressions revealed positive correlations between both joint regression coefficients and the mean height, $b_{0}$ (table 5 ).

Partial correlations between these three parameters showed that only the correlation between $\beta_{m}$ and $b_{0}$, was significant. That is, our attempt to correlate sensitivity for height $\left(\beta_{h}\right)$ with the parameters from equation (1) and (2) had failed. However, the significant correlation between $\beta_{m}$ and $b_{0}$ deserves comment for it emphasises the danger of drawing conclusions which our correlation matrix was not designed to show. We were explicitly trying to explain the variation within genotypes or the GE interaction for height in terms of age at flowering, and as such the parameters calculated by equations (1) and (2) related only to descriptions of height as a function of age at flowering, and not vice versa; that is, we can infer a relationship between some other measure of height such as $\beta_{h}$ and these parameters, but

TABLE 5

Correlations between coefficients from the regressions of final height in 10 Nicotiana genotypes

\begin{tabular}{|c|c|c|c|c|c|c|c|}
\hline \multirow[b]{3}{*}{ Equation } & \multirow{3}{*}{$\begin{array}{c}\text { Mean } \\
\text { final } \\
\text { height } \\
b_{0}\end{array}$} & \multicolumn{4}{|c|}{$\begin{array}{l}\text { Regressions of height on age } \\
\text { at flowering }\end{array}$} & \multirow{2}{*}{\multicolumn{2}{|c|}{$\begin{array}{c}\text { Joint } \\
\text { regressions } \\
\text { Equation (3) }\end{array}$}} \\
\hline & & \multicolumn{2}{|c|}{ Equation (1) } & \multicolumn{2}{|c|}{ Equation (2) } & & \\
\hline & & $b_{1}$ & $b_{2}$ & $c_{1}$ & $c_{2}$ & $\beta_{h}$ & $\beta_{m}$ \\
\hline (1) $\begin{array}{l}b_{0} \\
b_{1} \\
b_{2}\end{array}$ & & $\begin{array}{r}0.349 \\
-\end{array}$ & $\begin{array}{r}-0.043 \\
0.071 \\
-\end{array}$ & $\begin{array}{l}0 \cdot 355 \\
0 \cdot 455 \\
0 \cdot 239\end{array}$ & $\begin{array}{l}-0.705^{*} \\
-0.274 \\
-0.437\end{array}$ & $\begin{array}{c}0.740^{*} \\
0.098 \\
-0.176\end{array}$ & $\begin{array}{l}0 \cdot 886^{* * *} \\
0 \cdot 125 \\
-0 \cdot 221\end{array}$ \\
\hline (2) $\begin{array}{l}c_{1} \\
c_{2}\end{array}$ & & & & - & $\begin{array}{c}-0.619 \\
-\end{array}$ & $\begin{array}{r}0 \cdot 115 \\
-0.435\end{array}$ & $\begin{array}{r}0.154 \\
-0.497\end{array}$ \\
\hline (3) $\begin{array}{l}\beta_{h} \\
\beta_{m}\end{array}$ & & & & & & - & $\begin{array}{c}0 \cdot 791^{* *} \\
-\end{array}$ \\
\hline
\end{tabular}


not between a measure of age such as $\beta_{m}$ and these parameters. In fact, partial correlations taking into account the effects of mean age showed that the correlations between $\beta_{m}, \beta_{h}$ and $b_{0}$ were all non-significant, and that the partial correlation between $\beta_{m}$ and the mean age eliminating $\beta_{h}$ and $b_{0}$ was the only important relationship $(r=0.935, \mathrm{P}<0.001)$. This relationship would have been shown if we had calculated our matrix by regressing age on height to derive equations (1) and (2), in which case $b_{0}$ would have been the mean age at flowering.

There was also a significant negative correlation between mean height $\left(b_{0}\right)$ and $c_{2}$ from equation (2) (table 5). That is, it appeared that genotypes with greater mean height showed a proportionately greater decrease in height with later flowering, and that this relationship, because it derived from equation (2), was independent of the overall effects of differences between genotypes or environments. However, as with the correlations previously described for $\beta_{m}, \beta_{h}$ and $b_{0}$, partial correlations taking into account the effects of mean age showed that this correlation was not significant, and the only important relationship was between mean age and mean height $(r=0.877, \mathrm{P}<0.01)$.

Boughey and Jinks (1978) reported broadly similar results to these with the same variables measured in the same or similar Nicotiana genotypes in different experiments. They found that genotypic sensitivity for height (taken as the standard deviation of each genotype over a range of environments) tended to increase with flowering time and height at that time. It was particularly important, however, that they also found that the best predictor of sensitivity for height was the rate of increase in height of the different genotypes as measured by comparing height 6 weeks after transplanting with height at or after flowering.

\section{Discussion}

With few exceptions, when GE interactions have been described for crop plants, and when "useful " genetic variation for parameters of stable performance has been found, there has been no suggestion as to how the breeder can use this variation in his breeding programme. The ability to predict performance across environments (by using, for example, joint regression) is, of course, useful; but the problem remains of being able to select for stability or predictability of performance from segregating populations.

There are two obvious approaches to this problem. One is to select for stability on the basis of the performance of relatives in a range of environments, the other, which is far more commonplace, is to select genotypes with no account being taken of their stability of performance, and then screen them for stability. Brumpton et al. (1977) and Jinks et al. (1977) compared these two methods in Nicotiana, and found that family selection for stability was less efficient in producing genotypes with combinations of the required stability and mean performance than was the production of inbred lines by random single seed descent. They ascribed this to the unpredictable effects of seasons on the expression of stability in the relatives of the selected genotypes. In crops such as lettuce and cauliflower where, as in Nicotiana, single seed descent is easily realised, such a method of random inbreeding followed by screening is possible, but it would be impracticable 
if used as part of a breeding programme where several other characters were under simultaneous selection, for the amount of breeding material would become too large. Similar problems pertain to selection based on the replication of relatives in different environments.

An alternative is to define an environment in which particular phenotypes may be taken to represent genotypes which will give stability in a range of environments. Indeed, a generalisation has emerged from the work of Bateman (1971), Jinks and Connolly (1973, 1975) and Orozco (1976) that stability of good performance may be attained if the best performers in a poor environment are selected. One approach to this problem is that of Boughey and Jinks (1978) who measured the rate of increase in height in different genotypes, and successfully predicted the genotypic sensitivity of the same variable to environmental differences. We have attempted to define tests by which a particular type of environmental response to one variable may be taken to represent inherent stability to another variable. We have correlated parameters from joint regression with other measures of the plant phenotype using three examples where one variable could be regressed on another variable measured at about the same time. All of our examples involved chronological age as the independent variable and some measure of the floral state of the plant as the dependent variable (the cauliflower curd is floral tissue; lettuce is entering the floral stage when the head reaches marketability). These were chosen as being more likely to show correlations than variables with no obvious biological relationship.

Using our equation (2), that is, where genotypic and environmental effects were removed leaving only relationships caused by GE interactions, we could find no relationships with the results of joint regression or indeed with genotypic means for either of the variables in all three examples. However, the use of equation (1), that is, relationships depending on environmental effects within genotypes, showed interesting correlations with joint regression coefficients in lettuce and cauliflower, and with mean values for the variables in all three examples.

The results from the analysis in lettuce were illuminating in that positive correlations between high mean values and sensitivity to the environment for head weight were emphasised with the elimination of correlations with co-dependent parameters. A breeder faced with this situation would, logically, having tested this hypothesis, assess both the importance in commercial terms of high yielding lettuce being relatively unstable, and the possibility of selecting recombinants with high yield and stability following hybridisation of different genotypes in the hope that any linkages between genes controlling these variables might be broken.

In the cauliflower, our results show that stability for curd weight might be selected for by growing segregating populations in environments where weights were generally reduced due to a lengthened growing period. Our experience with this crop, including the data of Kesavan et al. (1976), suggests that crowding in the seedbed might afford just such a discriminating environment without unduly affecting the expression of other agronomic traits which must be simultaneously selected for. This result also goes some way towards explaining why a genotype selected as giving high curd weights at Wellesbourne, where good agronomic practices ensure a short growing period, gave much smaller curds when grown away from there under condi- 
tions where the growing period was longer (Crisp and Gray, 1978), and is, perhaps, a salutary tale for plant breeders.

We believe that these results have demonstrated the usefulness of correlating the sensitivity of genotypes (as defined by joint regression) with an unrelated variable. It seems probable to us that the technique is more likely to be useful if attempts are made to explain large GE interactions for a variable in terms of relatively small or at least controllable variation, which may include GE interactions, in a related variable, as we have done for lettuce and cauliflower. Attempts to explain GE interactions in terms of a related variable showing equivalently large or larger GE interactions may raise problems of cause and effect in the interpretation of the results, as occurred when we tried to explain GE interactions in Nicotiana. The approach taken by Boughey and Jinks (1978), of measuring the same variable, but at different times, may be of more use in these cases.

Acknowledgments.-We thank Professor J. L. Jinks of Birmingham University and our colleagues Drs V. Kesavan and D. Gray for permission to quote their data, and our NVRS colleagues Mr A. Barnes and Dr R. C. Hardwick for useful discussion.

\section{REFERENGES}

ARNOLD, M. H., AND innes, N. L. 1976. Plant breeding. In Agricultural Research for Development. The Namulonge Contribution, ed. M. H. Arnold. Cambridge University Press, 197-246.

BAteman, B. 1971. Selection of mice for growth on constant and changing maize-milk diets. Anim. Prod., 13, 425-440.

BOUGHEY, H., AND JINKs, J. L. 1978. Joint selection for both extremes of mean performance and of sensitivity to a macroenvironmental variable. III. The determinants of sensitivity. Heredity, 40, 363-369.

BRUMPTON, R. J., BOUGHEY, H., AND JINKS, J. L. 1977. Joint selection for both extremes of mean performance and of sensitivity to a macroenvironmental variable. I. Family selection. Heredity, 38, 219-226.

COCHRAN, w. G. 1957. Analysis of covariance: its nature and uses. Biometrics, 13, 261-281. CRISP, P., AND GRAY, A. R. 1978. Autumn cauliflower. Rep. natn. Veg. Res. Stn. for 1977, 25.

CRISP, P., JOHNSON, A. G., ELLIS, P. R., AND HARDMAN, J. A. 1977. Genetical and environmental interactions affecting resistance in radish to cabbage root fly. Heredity, 38, 209-218.

CRisP, P., AND KESAVAN, v. 1978. Genotypic and environmental effects on the weight of the curds of autumn-maturing cauliflowers. 7. agric. Sci., Camb., 90, 11-17.

DOWKER, B. D., JACKSON, J. C., AND FENNELL, J. F. M. 1976. Variation studies in carrots as an aid to breeding. IV. Associations between characters. $\mathcal{F}$. hort. Sci., 51, 245-252.

FINLAY, K. W., AND WILKINSON, G. N. 1963. The analysis of adaptation in a plant-breeding programme. Aust. 7. Agric. Res., 14, 742-754.

FREEMAN, G. H. 1973. Statistical methods for the analysis of genotype-environment interactions. Heredity, 31, 339-354.

FREEMAN, G. H., AND PERKINS, JEAN M. 1971. Environmental and genotype-environmental components of variability. VIII. Relations between genotypes grown in different environments and measures of these environments. Heredity, 27, 15-23.

FRIPP, Y. J. 1972. Genotype-environmental interactions in Schizophyllum commune. II. Assessing the environment. Heredity, 28, 223-238.

FRIPP, Y. J., AND CATEN, C. E. 1971. Genotype-environmental interactions in Schizophyllum commune. I. Analysis and character. Heredity, 27, 393-407.

GOODING, H. J., JENNINGS, D. L., AND TOPHAM, P. B. 1975. A genotype-environment experiment on strawberries in Scotland. Heredity, 34, 105-115.

HARDWICK, R. C., AND WOOD, J. T. 1972. Regression methods for studying genotypeenvironment interactions. Heredity, 28, 209-222.

HILL, J. 1975. Genotype-environment interactions-a challenge for plant breeding. 7. agric. Sci., Camb., 85, 477-493. 
JINKS, J. L., AND CONNOLLY, v. 1973. Selection for specific and general response to environmental differences. Heredity, 30, 33-40.

JINKS, J. L., AND CONNOLLY, v. 1975. Determination of the environmental sensitivity of selection lines by the selection environment. Heredity, 34, 401-406.

JINKS, J. L., JAYASEKARA, N. E. M., AND BOUGMEY, MILARIE. 1977. Joint selection for both extremes of mean performance and of sensitivity to a macroenvironmental variable. II. Single seed descent. Heredity, 39, 345-355.

Kendali, M. G., AND STUART, A. 1973. The Advanced Theory of Statistics, Vol. 2, 3rd edition. Charles Griffin \& Co., London.

KESAVAN, V., CRISP, P., GRAY, A. R., AND DOWKER, B. D. 1976. Genotypic and environmental effects on the maturity time of autumn cauliflowers. Theoret. Appl. Genetics, 47, 133-140.

KNIGHT, R. 1970. The measurement and interpretation of genotype-environment interactions. Euphytica, 19, 225-235.

OROzCO, F. 1976. A dynamic study of genotype-environment interaction with egg laying of Tribolium castaneum. Heredity, 37, 157-171.

PERKINS, J. M., AND JINKS, J. L. 1968. Environmental and genotype-environmental components of variability. III. Multiple lines and crosses. Heredity, 23, 339-356.

PERKINS, J. M., AND JINKS, J. L. 1973. The assessment and specificity of environmental and genotype-environmental components of variability. Heredity, 30, 111-126.

WOOD, J. T. 1976. The use of environmental variables in the interpretation of genotypeenvironment interaction. Heredity, 37, 1-7.

yates, F., AND COCMRAN, W. G. 1938. The analysis of groups of experiments. F. agric. Sci., Camb., 28, 556-580. 